\title{
ERG is specifically associated with ETS-2 and ETV-4, but not with ETS-1, in prostate cancer
}

\author{
DAVID ADLER ${ }^{1}$, JACQUELINE OCHSENFAHRT $^{1}$, KERSTIN FUCHS $^{1}$, \\ GLEN KRISTIANSEN $^{1}$, SVEN PERNER $^{1,2}$ and NICOLAS WERNERT ${ }^{1}$ \\ ${ }^{1}$ Institute of Pathology and ${ }^{2}$ Department of Prostate Cancer Research, Institute of Pathology, \\ University Hospital of Bonn, D-53127 Bonn, Germany
}

Received May 8, 2012; Accepted June 22, 2012

DOI: $10.3892 /$ ijmm.2012.1097

\begin{abstract}
The erythroblast transformation-specific (ETS) family of transcription factors plays important roles in both physiological and pathological conditions. Even though many studies have focused on single ETS factors within a single tissue and within the context of specific promoters, the functional impact of multiple ETS members present within a specific cell type has not yet been investigated, especially in prostate cancer $(\mathrm{PCa})$. As the most prominent gene rearrangement in PCa leads to the overexpression of the ETS-related gene (ERG), the aim of this study was to investigate whether ERG is part of a complex integrated transcriptional network that involves other ETS factors. More specifically, as the ETS family consists of 27 members, we focused our efforts initially on investigating whether ERG is associated with the three family members, ETS-1, ETS-2 and ETS variant gene-4 (ETV-4), in PCa as a proof of principle. Using western blot analysis, we show that ERG, ETS-1, ETS-2 and ETV-4 are expressed in PC3 cell nuclear extracts and in protein lysates prepared from human PCa prostatectomy specimens. Immunoprecipitations using an anti-ERG antibody were used with PC3 cell nuclear extracts as well as with a pooled protein lysate sample prepared from the PCa tissue samples of five patients. Importantly, our results revealed that ERG is specifically associated with ETS-2 and ETV-4, but not with ETS-1, in PC3 cell nuclear extracts and PCa tissue protein lysates. Our findings strongly support the notion that ERG is part of a complex integrated transcriptional network that involves other ETS factors, which are likely to cooperate or influence the activity of ERG in PCa. The functional impact of multiple ETS factors being associated with ERG in PCa requires further study, as it may provide insights into the mechanism by
\end{abstract}

Correspondence to: Professor Nicolas Wernert, Institute of Pathology, University Hospital of Bonn, 25 Sigmund-Freud St., D-53127 Bonn, Germany

E-mail: nicolas.wernert@ukb.uni-bonn.de

Key words: ETS-related gene, ETS-2, ETS variant gene-4, ETS-1, prostate cancer which ERG exerts its influence in PCa and may subsequently contribute to our understanding of the molecular basis of PCa.

\section{Introduction}

Prostate cancer ( $\mathrm{PCa}$ ) is a leading cause of cancer mortality and is among the most common tyeps of cancer in Western countries (1). In PCa, recurrent gene fusions occurring between the transmembrane protease, serine 2 (TMPRSS2) gene, an androgen-regulated prostate-specific serine protease and members of the erythroblast transformation-specific (ETS) family of transcription factors [ETS variant gene (ETV)-1, ETV-4, ETV-5 and most commonly, ETS-related gene ERG] resulting in the increased expression of the latter rearranged ETS members in response to androgens, are frequently found (2-4).

The ETS family of transcription factors which is comprised of 27 members in humans (5) has been reported to be involved in many processes such as development, differentiation, proliferation, apoptosis, migration, tissue remodeling, invasion and angiogenesis in a wide range of cells (such as B-cells, endothelial cells, fibroblasts, as well as neoplastic cells) (6-13). The ETS family is defined by the presence of an evolutionary conserved DNA-binding domain, termed the ETS domain, which is comprised of approximately 80 amino acids with four tryptophan repeats and recognizes DNA sequences with a GGAA/T core motif $(14,15)$. Phylogenetic analysis of the human ETS domains has led to the identification of subfamilies of more highly related members (16).

Even though different ETS family members may have different functions due to their binding preferences for distinct flanking sequences around the GGAA/T core motif enabling them to bind more specifically (16), overlapping functions of ETS members and redundant occupancy at gene regions have been described $(16,17)$. Furthermore, even though the ETS transcription factors may have independent activities, it is likely that they are part of an integrated network, in which gene regulation may be influenced by the binding equilibrium and the activity of the ETS transactivation domains, as well as the complex formation of different ETS members and other factors $(18,19)$.

While a number of studies have focused on single ETS factors within a single tissue and within the context of specific promoters, the functional impact of multiple ETS members 
present within a specific cell type have not yet been investigated (18), particularly in PCa. While multiple ETS factors may be able to regulate the same sets of genes, the magnitude or the directions may be different for each of the ETS factors (18).

As the most prominent gene rearrangement in PCa leads to the overexpression of ERG (20-23), combined with functional studies showing that $E R G$ knockdown induces morphological changes, inhibits cell growth in both culture and mice, and that $E R G$ overexpression leads to an increase in cell invasion (24), the aim of the present study was to investigate whether ERG is part of a complex integrated transcriptional network that involves other ETS factors which are highly likely to cooperate or influence the activity of ERG in PCa.

More specifically, as the ETS family of transcription factors consists of 27 members (5), we decided to focus our efforts initially on investigating whether ERG is associated with three well-known members of the family, ETS-1, ETS-2 and ETV-4, in PCa as a proof of principle. The rationale behind choosing the latter ETS members was that ETS-1, the prototype of the ETS family, is overexpressed in latent as well as clinically manifest PCa (25), ETS-2 is also overexpressed in PCa (18), ETS-2 and ETS-1 play redundant roles (17), ETS-2 is associated with in vitro synthesized ETS-1 (26), ETS-2 interacts with ERG in vivo demonstrated by the two-hybrid system (26) and that ETV-4 is rearranged in PCa, similar to ERG (2-4). The results from a previous study were also taken into account, namely that the occurrence of multiple ETS rearrangements within one prostate gland, within the same tumor focus and within the same nucleus (27).

\section{Materials and methods}

Western blot analysis. The expression of ERG, ETS-1, ETS-2 and ETV-4 in PC3 cell nuclear extracts (Santa Cruz Biotechnology, Inc., Santa Cruz, CA, USA) was determined by western blot analysis using a mouse monoclonal anti-ERG antibody (Bio-Care, Holt, MI, USA), a mouse monoclonal antiETS-1 antibody (Transduction Laboratories, Lexington, KY, USA), a rabbit polyclonal anti-ETS-2 antibody (Sigma-Aldrich, Munich, Germany) and a mouse monoclonal anti-ETV-4 antibody (BioCat, Heidelberg, Germany), respectively.

In protein lysates prepared from human PCa prostatectomy specimens of five patients, the expression of ERG, ETS-1, ETS-2 and ETV-4 was determined by western blot analysis using a mouse monoclonal anti-ERG antibody (Biocare), a mouse monoclonal anti-ETS-1 antibody (Transduction Laboratories) and a rabbit polyclonal C-20 anti-ETS-1 antibody (Santa Cruz Biotechnology, Inc.), a rabbit polyclonal anti-ETS-2 antibody (Sigma-Aldrich) and a mouse monoclonal anti-ETV-4 antibody (BioCat), respectively.

Human PCa prostatectomy specimens and protein lysate preparations. Briefly, fresh tissue samples from five patients with prostate carcinomas (Gleason scores, 6, 6 7,7 and 8) were taken immediately after radical prostatectomy. The tissue samples were then shock-frozen in liquid nitrogen with ice-cold isopentane as described previously (28). Thereafter, $6-\mu \mathrm{m}$-thick frozen sections were cut from the samples using a cryotome (Leica, Berlin, Germany) and mounted on conventional slides followed by staining with hematoxylin and eosin (H\&E) for diagnostic evaluation by an experienced pathologist. The preparation of protein lysates from the latter prostate carcinoma tissue samples was carried out as previously described (29).

Immunoprecipitation (IP). To investigate whether ERG is associated with ETS-1, ETS-2 and ETV-4 in PC3 cell nuclear extracts (Santa Cruz Biotechnology, Inc.), we performed IP using a rabbit polyclonal anti-ERG antibody (Gentex, Zeeland, MI, USA) as it exhibited the best compatibility with our IP compared to all the other commercially available antibodies that we tested. Briefly, PC3 cell nuclear extracts were precleared with protein A agarose beads (Sigma-Aldrich) by rotation at $4^{\circ} \mathrm{C}$ for $2 \mathrm{~h}$. An additional tube containing protein $\mathrm{A}$ agarose beads (Sigma-Aldrich) and the rabbit polyclonal anti-ERG antibody (Gentex) was incubated by rotation at $4^{\circ} \mathrm{C}$ for $2 \mathrm{~h}$. Both tubes were then centrifuged at 2,000 x $\mathrm{g}$ for 2 min and the pre-cleared supernatant was then added to the ERG-bound protein A agarose beads. The sample was then incubated by rotation for $1 \mathrm{~h}$ at $4^{\circ} \mathrm{C}$, collected by centrifugation at $2,000 \mathrm{x} \mathrm{g}$ for $2 \mathrm{~min}$, and then washed three times with a nuclear extract buffer (20 mM Hepes, $25 \%$ glycerol, $2 \mathrm{M} \mathrm{KCL}$, $1 \mathrm{mM} \mathrm{MgCl}$, $1 \%$ Nonidet-p40, $0.5 \mathrm{mM}$ EDTA). The beads were then re-suspended in a loading buffer, boiled and loaded on an SDS/PAGE gel, followed by western blot analysis using a mouse monoclonal anti-ERG antibody (Biocare), a mouse monoclonal anti-ETS-1 antibody (Transduction Laboratories), a rabbit polyclonal anti-ETS-2 antibody (Sigma-Aldrich) and a mouse monoclonal anti-ETV-4 antibody (BioCat), respectively.

In order to investigate whether ERG is associated with ETS-1, ETS-2 and ETV-4 in the protein lysates prepared from the above five PCa prostatectomy specimens, we first pooled all the five protein lysates and then performed IP as described above. Western blot analysis was performed with the same antibodies used for the above IP and additionally with a rabbit polyclonal C-20 anti-ETS-1 antibody (Santa Cruz Biotechnology, Inc.).

\section{Results}

Protein expression of ERG, ETS-1, ETS-2 and ETV-4 in PC3 cell nuclear extracts and in protein lysates prepared from human PCa prostatectomy specimens. As shown by western blot analysis using specific antibodies, ERG, ETS-1, ETS-2 and ETV-4 were expressed in PC3 cell nuclear extracts (Santa Cruz Biotechnology, Inc.), as well as in protein lysates prepared from the human $\mathrm{PCa}$ prostatectomy specimens of five patients (Fig. 1).

ERG is specifically associated with ETS-2 and ETV-4, but not with ETS-1, in PC3 cell nuclear extracts. To investigate whether ERG is associated with ETS-1, ETS-2 and ETV-4 in PC3 cell nuclear extracts (Santa Cruz Biotechnology, Inc.), IP was performed using a rabbit polyclonal anti-ERG antibody (Gentex). Following western blot analysis, the results revealed that ERG is specifically associated with ETS-2 and ETV-4, but not with ETS-1 (Fig. 2).

ERG is specifically associated with ETS-2 and ETV-4, but not with ETS-1, in protein lysates prepared from human PCa 

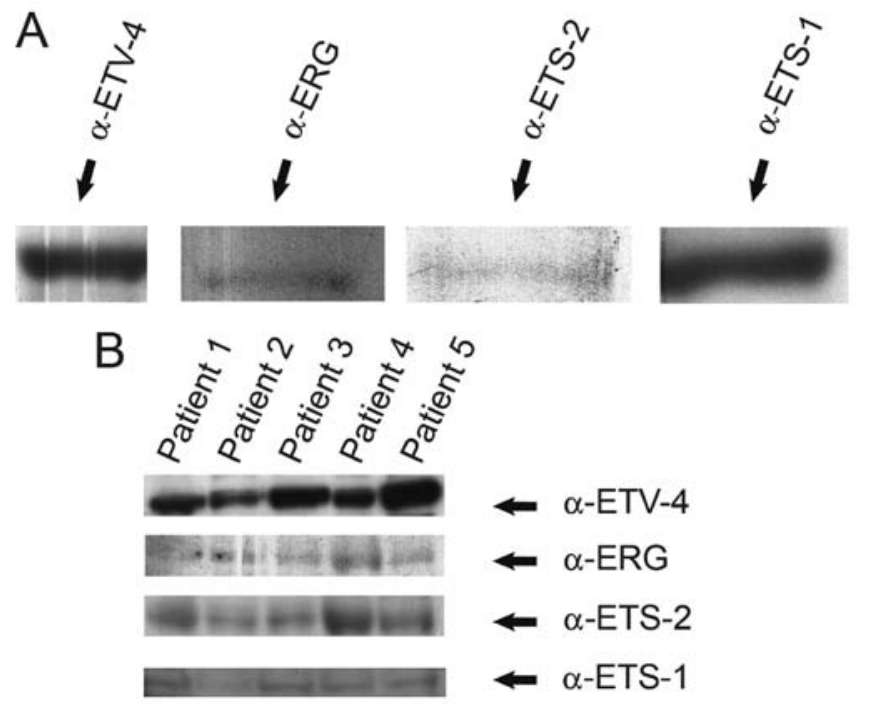

Figure 1. Expression of ERG, ETS-1, ETS-2 and ETV-4 in PC3 cell nuclear extracts and PCa tissues. Western blot analysis showed that ERG, ETS-1, ETS-2 and ETV-4 were expressed in (A) PC3 cell nuclear extracts, as well as in protein lysates prepared from (B) the PCa tissue samples of five patients.

A
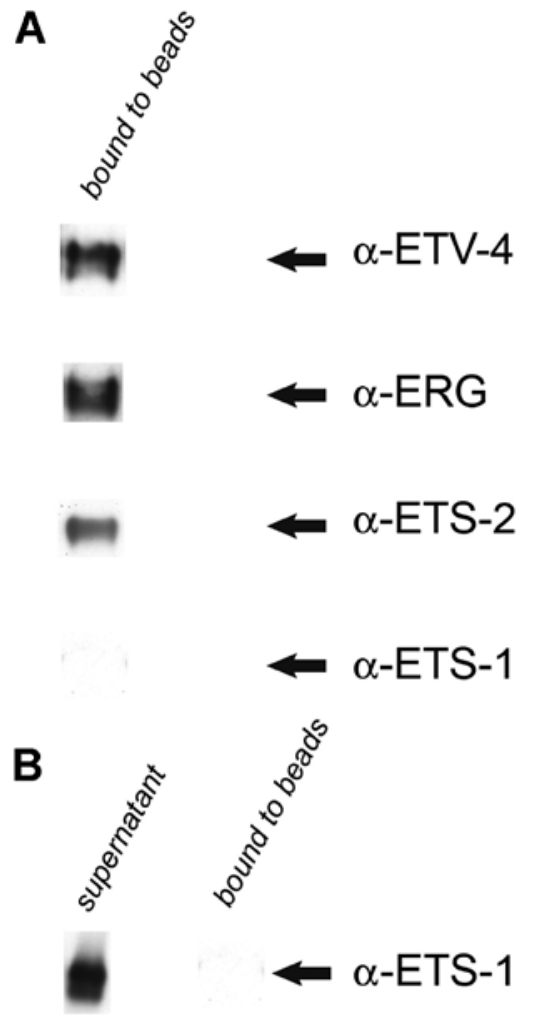

Figure 2. ERG is specifically associated with ETS-2 and ETV-4, but not with ETS-1, in PC3 cell nuclear extracts. Immunoprecipitation (IP) using an antiERG antibody showed that ERG is specifically associated with (A) ETS-2 and ETV-4, but not with (B) ETS-1, in PC3 cell nuclear extracts.

prostatectomy specimens. Using IP with a rabbit polyclonal anti-ERG antibody (Gentex), we investigated whether ERG is associated with ETS-1, ETS-2 and ETV-4 in a pooled protein lysate sample that was prepared from the PCa prostatectomy specimens of five patients. Following western blot analysis,
A
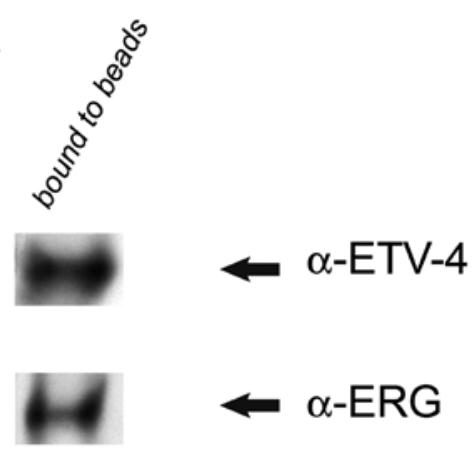

$\leftarrow \alpha$-ERG

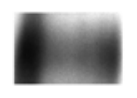

$\leftarrow \alpha$-ETS-2

$\alpha$-ETS-1

B

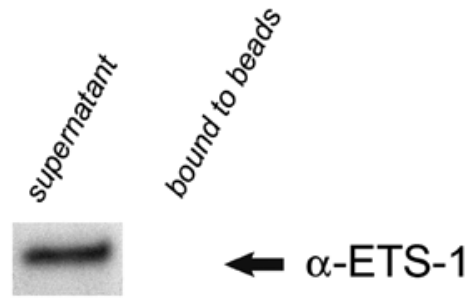

Figure 3. ERG is specifically associated with ETS-2 and ETV-4, but not with ETS-1, in PCa tissues. Immunoprecipitation (IP) using an anti-ERG antibody showed that ERG is specifically associated with (A) ETS-2 and ETV-4, but not with (B) ETS-1, in a pooled protein lysate sample prepared from the $\mathrm{PCa}$ tissue samples of five patients.

the results revealed that ERG is specifically associated with ETS-2 and ETV-4, but not with ETS-1 (Fig. 3).

\section{Discussion}

The most prominent gene rearrangement in $\mathrm{PCa}$ arises between the androgen-regulated prostate-specific serine protease $T M P R S S 2$ gene and the ETS transcription factor $E R G$ gene, leading to the overexpression of ERG (20-23). The ERG rearrangement is highly specific to $\mathrm{PCa}(30)$, is maintained in advanced disease $(2,31)$, and depending on the cohort design and the histological subtypes of PCa $(20,32-34)$ approximately $15-80 \%$ of PCa patients harbour the TMPRSS2-ERG fusions $(2-4,35,36)$. Furthermore, the knockdown of $E R G$ has been reported to induce morphological changes and the inhibition of cell growth in both cell cultures and mice, whereas $E R G$ overexpression has been shown to result in an increase in cell invasion (24).

Even though ETS transcription factors, such as ERG may have independent activities in PCa, ERG is highly likely to be part of a complex integrated transcriptional network that involves other ETS factors. In such a network, gene regulation may be influenced by the binding equilibrium, the activity of ETS transactivation domains and a complex formation of different ETS members and other factors, and even if multiple ETS factors may be able to regulate the same sets of genes, 
the magnitude or the directions may be different for each of the ETS factors $(18,19)$.

While the focus of many studies has been on single ETS factors within a single tissue and within the context of specific promoters, the functional impact of multiple ETS factors present within a specific cell type has not yet been well investigated (18), particularly in PCa. Therefore, the aim of this study was to investigate whether ERG is associated with specific ETS transcription factors in PCa. More specifically, we investigated whether ERG is associated with ETS-1, ETS-2 and ETV-4 in PC3 cell nuclear extracts, as well as in PCa tissues.

As the ETS family of transcription factors consists of 27 members in humans (5), we decided to focus our efforts initially on investigating whether is ERG associated with three well-known members of the family, ETS-1, ETS-2 and ETV-4, in PCa as a proof of principle. The rationale behind choosing the latter ETS members was as follows: ETS-1 is the prototype of the ETS family and has been reported to be overexpressed in latent as well as clinically manifest PCa and a strong expression of ETS-1 has been associated with poor tumor differentiation (25). As shown in a previous study of our, the blockade of ETS-1 in PCa cell lines results in a decrease in cell migration (11) and has a major effect upon genes involved in the metastatic cascade (12). As ETS-1 and ETS-2 have been reported to play redundant roles during embryonic development (17), combined with reports that ETS-2 is associated with in vitro synthesized ETS-1 (26) and that ETS-2 interacts with ERG in vivo demonstrated by the two-hybrid system (26), we decided to investigate ETS-2 as well. ETS-2 has been reported to be overexpressed in PCa (18), and a blockade of ETS-2 has been shown to reduce the transformed properties of $\mathrm{PCa}$ cells (37), as well as growth inhibition and apoptosis (38). ETV-4 on the other hand, was chosen as ETV-4 is known to be rearranged in PCa, similar to ERG (2-4), combined with a report showing the occurrence of multiple ETS rearrangements within one prostate gland, within the same tumor focus and within the same nucleus (27). Moreover, we recently reported the ETV-4 rearranged gene status in primary PCas and their corresponding lymph nodes, and found the rearrangement in $6 \%$ of both primary PCas and the corresponding lymph node metastases (39). Furthermore, ETV-4 has been shown to be required for anchorage-independent growth and cell proliferation gene expression program in PCa cell lines (40).

Prior to investigating whether is ERG associated with ETS-1, ETS-2 and ETV-4, we first examined whether these latter proteins are expressed in PC3 cell nuclear extracts and in protein lysates prepared from human PCa prostatectomy specimens. We found by western blot analysis that ERG, ETS-1, ETS-2 and ETV-4 are expressed in both PC3 cell nuclear extracts as well as in protein lysates prepared from the PCa tissue samples of five patients (Fig. 1).

Upon confirming the expression of ERG, ETS-1, ETS-2 and ETV-4 (Fig. 1), we performed IP using an anti-ERG antibody to investigate whether ERG is associated with ETS-1, ETS-2 and ETV-4 in PC3 cell nuclear extracts, as well as in a pooled protein lysate sample prepared from the PCa tissue samples of five patients.

Of note, our results revealed that ERG is specifically associated with ETS-2 and ETV-4, but not with ETS-1, in PC3 cell, nuclear extracts (Fig. 2) and even more remarkably, we found ERG to exhibit the same specificity for ETS-2 and ETV-4, but not for ETS-1 in the pooled protein lysate sample prepared from the PCa tissue samples of five patients (Fig. 3).

Our observation that ERG is not associated with ETS-1 and ETS-2, but rather only with ETS-2, may be due to the fact that ETS-1 and ETS-2 have been reported to play redundant roles (17), which would make it counterproductive for ERG to be associated with ETS-1 and ETS-2 simultaneously. The association of ERG with ETS-2 based on our findings is supported by a previous study reporting that ERG interacts with ETS-2 in vivo using the two-hybrid system (26). Our finding that ERG is associated with ETV-4 is highly intriguing, as ETV-4 is known to be rearranged in PCa, similar to $E R G(2-4,39)$, combined with a recent report showing the occurrence of multiple ETS rearrangements within one prostate gland, within the same tumor focus and within the same nucleus (27), which may imply a potential cooperation or interaction between rearranged ETS genes in PCa.

Taken together, our findings that ERG is specifically associated with ETS-2 and ETV-4, but not with ETS-1, in PC3 cell nuclear extracts and PCa tissues strongly support the notion that ERG is part of a complex integrated transcriptional network that involves other ETS factors, such as ETS-2 and ETV-4, which are likely to cooperate or influence the activity of ERG in PCa. The functional impact of multiple ETS factors associating with ERG in PCa should be investigated in further studies as it may provide insights into the mechanism in which ERG exerts its influence in PCa, and subsequently contribute to our understanding of the molecular basis of PCa.

\section{Acknowledgements}

The present study was supported by a grant from the German Research Foundation [Deutsche Forschungsgemeinschaft (DFG)] (WE1104/11-1) and the German Cancer Aid (Deutsche Krebshilfe, 107827) to N.W. and by a grant from the German Research Foundation [Deutsche Forschungsgemeinschaft (DFG)], Emmy-Noether-Program, (PE1179/2-1), the RudolfBecker-Foundation and the Wilhelm-Sander-Foundation (2011.077.1) to S.P.

\section{References}

1. Jemal A, Siegel R, Xu J and Ward E: Cancer statistics, 2010. CA Cancer J Clin 60: 277-300, 2010.

2. Tomlins SA, Rhodes DR, Perner S, et al: Recurrent fusion of TMPRSS2 and ETS transcription factor genes in prostate cancer. Science 310: 644-648, 2005.

3. Rubin MA, Maher CA and Chinnaiyan AM: Common gene rearrangements in prostate cancer. J Clin Oncol 29: 3659-3668, 2011.

4. Kumar-Sinha C, Tomlins SA and Chinnaiyan AM: Recurrent gene fusions in prostate cancer. Nat Rev Cancer 8: 497-511, 2008.

5. Hollenhorst PC, Jones DA and Graves BJ: Expression profiles frame the promoter specificity dilemma of the ETS family of transcription factors. Nucleic Acids Res 32: 5693-5702, 2004

6. Oikawa T: ETS transcription factors: possible targets for cancer therapy. Cancer Sci 95: 626-633, 2004.

7. Hashiya N, Jo N, Aoki M, et al: In vivo evidence of angiogenesis induced by transcription factor Ets-1: Ets-1 is located upstream of angiogenesis cascade. Circulation 109: 3035-3041, 2004.

8. Rothhammer T, Hahne JC, Florin A, et al: The Ets-1 transcription factor is involved in the development and invasion of malignant melanoma. Cell Mol Life Sci 61: 118-128, 2004. 
9. Hahne JC, Okuducu AF, Kaminski A, Florin A, Soncin F and Wernert N: Ets-1 expression promotes epithelial cell transformation by inducing migration, invasion and anchorage-independent growth. Oncogene 24: 5384-5388, 2005.

10. Sahin A, Vercamer C, Kaminski A, et al: Dominant-negative inhibition of Ets 1 suppresses tumor growth, invasion and migration in rat C6 glioma cells and reveals differentially expressed Ets 1 target genes. Int J Oncol 34: 377-389, 2009.

11. Adler D, Langer B, Lindstrot A,et al: Ets-1 is implicated in the regulation of androgen co-regulator FHL2 and reveals specificity for migration, but not invasion, of PC3 prostate cancer cells. Oncol Rep 25: 1125-1129, 2011.

12. Adler D, Lindstrot A, Langer B, Buettner $R$ and Wernert N: Comprehensive gene expression microarray analysis of Ets-1 blockade in PC3 prostate cancer cells and correlations with prostate cancer tissues: Insights into genes involved in the metastatic cascade. Int J Mol Med 27: 811-819, 2011.

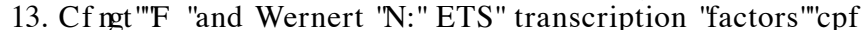
prostate cancer: The role of the family prototype ETS-1 (Review). Int J Oncol 40: 1748-1754, 2012.

14. Dittmer J: The biology of the Ets1 proto-oncogene. Mol Cancer 2: 29,2003

15. Wasylyk B, Hahn SL and Giovane A: The Ets family of transcription factors. Eur J Biochem 211: 7-18, 1993.

16. Hollenhorst PC, Shah AA, Hopkins C and Graves BJ: Genomewide analyses reveal properties of redundant and specific promoter occupancy within the ETS gene family. Genes Dev 21 1882-1894, 2007.

17. Wei G, Srinivasan R, Cantemir-Stone CZ, et al: Ets1 and Ets2 are required for endothelial cell survival during embryonic angiogenesis. Blood 114: 1123-1130, 2009.

18. Watson DK, Turner DP, Scheiber MN, Findlay VJ and Watson PM: ETS transcription factor expression and conversion during prostate and breast cancer progression. Open Cancer J 3 : 24-39, 2010.

19. Wei GH, Badis G, Berger MF, et al: Genome-wide analysis of ETS-family DNA-binding in vitro and in vivo. EMBO J 29: 2147-2160, 2010

20. Tomlins SA, Bjartell A, Chinnaiyan AM, et al: ETS gene fusions in prostate cancer: from discovery to daily clinical practice. Eur Urol 56: 275-286, 2009.

21. Petrovics G, Liu A, Shaheduzzaman S, et al: Frequent overexpression of ETS-related gene-1 (ERG1) in prostate cancer transcriptome. Oncogene 24: 3847-3852, 2005.

22. Rostad K, Mannelqvist M, Halvorsen OJ, et al: ERG upregulation and related ETS transcription factors in prostate cancer. Int J Oncol 30: 19-32, 2007.

23. Cerveira N, Ribeiro FR, Peixoto A, et al: TMPRSS2-ERG gene fusion causing ERG overexpression precedes chromosome copy number changes in prostate carcinomas and paired HGPIN lesions. Neoplasia 8: 826-832, 2006.

24. Sun C, Dobi A, Mohamed A, et al: TMPRSS2-ERG fusion, a common genomic alteration in prostate cancer activates C-MYC and abrogates prostate epithelial differentiation. Oncogene 27 : $5348-5353,2008$.
25. Alipov G, Nakayama T, Ito M, et al: Overexpression of Ets-1 proto-oncogene in latent and clinical prostatic carcinomas. Histopathology 46: 202-208, 2005.

26. Basuyaux JP, Ferreira E, Stehelin D and Buttice G: The Ets transcription factors interact with each other and with the c-Fos/c-Jun complex via distinct protein domains in a DNA-dependent and -independent manner. J Biol Chem 272: 26188-26195, 1997.

27. Svensson MA, LaFargue CJ, MacDonald TY, et al: Testing mutual exclusivity of ETS rearranged prostate cancer. Lab Invest 91: 404-412, 2011.

28. Adler D, Lindstrot A, Ellinger J, Rogenhofer S, Buettner R and Wernert N: Identification of immunity-related genes in prostate cancer and potential role of the ETS family of transcription factors in their regulation. Int J Mol Med 28: 799-807, 2011.

29. Espina V, Wulfkuhle JD, Calvert VS, et al: Laser-capture microdissection. Nat Protoc 1: 586-603, 2006.

30. Scheble VJ, Braun M, Beroukhim R, et al: ERG rearrangement is specific to prostate cancer and does not occur in any other common tumor. Mod Pathol 23: 1061-1067, 2010.

31. Clark JP and Cooper CS: ETS gene fusions in prostate cancer. Nat Rev Urol 6: 429-439, 2009.

32. Han B, Mehra R, Suleman K, et al: Characterization of ETS gene aberrations in select histologic variants of prostate carcinoma. Mod Pathol 22: 1176-1185, 2009.

33. Guo CC, Dancer JY, Wang Y, et al: TMPRSS2-ERG gene fusion in small cell carcinoma of the prostate. Hum Pathol 42: 11-17, 2011.

34. Scheble VJ, Braun M, Wilbertz T, et al: ERG rearrangement in small cell prostatic and lung cancer. Histopathology 56: 937-943, 2010.

35. Joshua AM, Evans A, Van der Kwast T, et al: Prostatic preneoplasia and beyond. Biochim Biophys Acta 1785: 156-181, 2008.

36. Braun M, Scheble VJ, Menon R, et al: Relevance of cohort design for studying the frequency of the ERG rearrangement in prostate cancer. Histopathology 58: 1028-1036, 2011.

37. Sementchenko VI, Schweinfest CW, Papas TS and Watson DK: ETS2 function is required to maintain the transformed state of human prostate cancer cells. Oncogene 17: 2883-2888, 1998.

38. Carbone GM, Napoli S, Valentini A, Cavalli F, Watson DK and Catapano CV: Triplex DNA-mediated downregulation of Ets2 expression results in growth inhibition and apoptosis in human prostate cancer cells. Nucleic Acids Res 32: 4358-4367, 2004

39. Shaikhibrahim Z, Braun M, Nikolov P, et al: Rearrangement of the ETS genes ETV-1, ETV-4, ETV-5, and ELK-4 is a clonal event during prostate cancer progression. Hum Pathol: May 7 , 2012 (Epub ahead of print).

40. Hollenhorst PC, Paul L, Ferris MW and Graves BJ: The ETS gene ETV4 is required for anchorage-independent growth and a cell proliferation gene expression program in PC3 prostate cells. Genes Cancer 1: 1044-1052, 2011. 